\title{
Volume discrimination of nanoparticles via electrical trapping using nanopores
}

\author{
Akihide Arima* ${ }^{*}$, Makusu Tsutsui ${ }^{*}$ and Masateru Taniguchi
}

\begin{abstract}
Electrophoretic capture of an oversized object on a solid-state nanopore is a useful approach for single-particle analyses via post electrical and optical measurements. Here we report on nanoparticle discriminations by the volume through combining this nanopore trap method with the cross-membrane ionic current measurements. We investigated ion transport through a pore channel being partially occluded by an electrophoretically-drawn nanoparticle at the orifice. We found distinct difference in the amount of current blockage by particles of different sizes. Multiphysics simulations revealed dominant contribution of particle volume over the other properties. We also demonstrated single-particle discriminations of two different sizes in a mixture solution. The present results demonstrate that this electrical capturing is a promising technique to immobilize a target at a single particle level that concomitantly offer wealth of information concerning their volume.
\end{abstract}

Keywords: Nanopore, Electrophoresis, Biosensor, Resistive pulse measurement

\section{Introduction}

Nanopore analyses are a simple and strong method for a particle characterization that enables evaluations of various parameters such as the shape, volume, and surface charge density [1-8]. In the measurement, electrophoretic entering of analytes into the nanoscale conduit causes a short-time decreasing of the cross-pore ionic current, and the associated blockade current is used for studying the physical characteristics of individual analytes [9-12]. This method, however, cannot be used for repetitive measurements of single-particles unless additional probes are incorporated to regulate the fast translocation motions such as dielectrophoresis [13], optical tweezer [14], and a magnetic force [15].

On the other hand, nanopore trap method is a more simple and facile strategy for the target immobilization at a single-particle level (Fig. 1a) [16, 17]. This method utilizes a nanopore with a diameter smaller than that of analytes of interest. Unlike conventional resistive pulse measurements [18], the targets are not able to pass

\footnotetext{
*Correspondence: arima@sanken.osaka-u.ac.jp; tsutsui@sanken.osaka-u. ac.jp

The Institute of Scientific and Industrial Research, Osaka University, 8-1
} Mihogaoka, Ibaraki, Osaka 567-0047, Japan through the pore but become immobilized at the orifice under the applied electrophoretic voltage. Previous works [16] have proven the ability of discrimination between surface charges of equi-sized nanoparticles using a low thickness-to-diameter aspect ratio nanopore. Here, in this report, we investigated the feasibility of the nanopore trap method for discriminating particles by the volume. We repetitively measured the ionic current blockage of two nanoparticles having different sizes. As a result, larger particles were found to block the ion transport more effectively whereby enabled discriminations of single-particles by the volume. This finding can open the prospect for tracing target condition to gain wealthy information about the trapped analyte such as in the situation of cell growth and shows the advantage in the incorporation of additional probes such as tunneling current via nanoelectrode employing capability of the method as a delivery and capture system [19-22].

\section{Methods}

The fabrication process of a nanopore is described elsewhere [8, 17]. Briefly, $20 \mathrm{~mm} \times 20 \mathrm{~mm}$ sized silicon chips constructed with three layers, $\mathrm{SiN} / \mathrm{Si} /$ $\mathrm{SiN}=50 \mathrm{~nm} / 0.5 \mathrm{~mm} / 50 \mathrm{~nm}$, were used as substrates. Through a reactive ion etching (RIE) for removing 


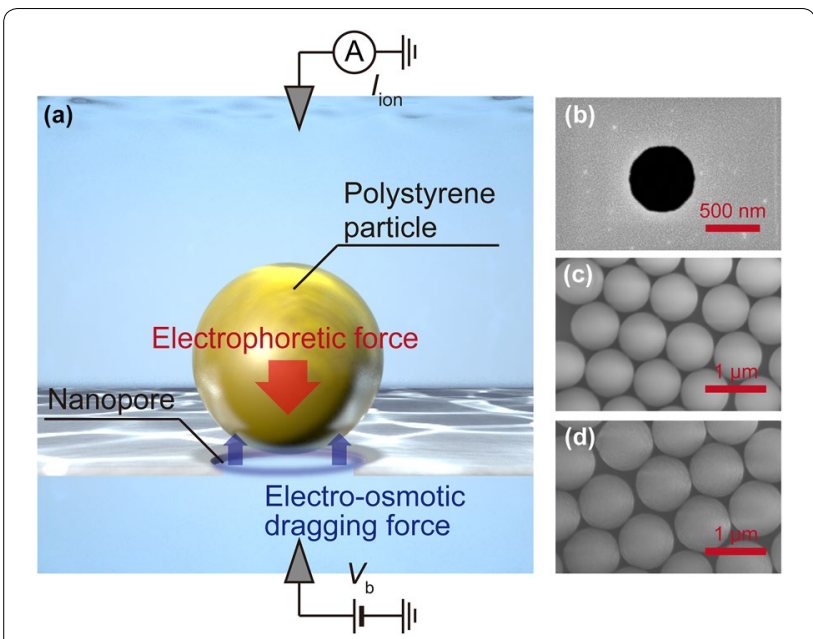

Fig. 1 a Schematic image of nanopore trapping method and $\mathbf{b}-\mathbf{d}$ scanning microscopic images of pore and particles. b A $600 \mathrm{~nm}$-sized nanopore was employed for capturing carboxylated-polystyrene particles sized $\mathbf{c} 780 \mathrm{~nm}$ and $\mathbf{d} 900 \mathrm{~nm}$

partial area of $\mathrm{SiN}$ layer on one side of the surfaces and following anisotropic wet etching of $\mathrm{Si}$ in $\mathrm{KOH}$ aq., a $50 \mathrm{~nm}$-thick SiN membrane was prepared. After forming metal patterns (thickness: $\mathrm{Cr} / \mathrm{Au}=2 \mathrm{~nm} / 30 \mathrm{~nm}$ ) by photolithography, radio-frequency magnetron sputtering, and lift off in $N, N$-dimethylformamide, a $600 \mathrm{~nm}$-sized pore was excavated using electron-beam lithography and RIE (Fig. 1b) in the thin membrane through using the metal patterns as markers.

For the nanopore measurements, polydimethylsiloxane (PDMS) blocks having microchannels were attached on both sides of the chip. Suspension of target particles was then injected through inlet and outlet holes penetrated in the polymeric blocks. After the injection, $\mathrm{Ag} / \mathrm{AgCl}$ electrodes were set on the both blocks for application of the electrophoretic voltage $V_{\mathrm{b}}$ and measuring the ionic current $I_{\text {ion }}$ using Keithley 6487 picoammeter/source (Tektronix, Inc.) under the particle trap control by the handmade program using Visual Basic 6.0.

As target analyte, two carboxylated-polystyrene particles (PS-COOH) with diameter $780 \mathrm{~nm}$ and $900 \mathrm{~nm}$ (Fig. 1c, d, Thermo Fisher Scientific, Inc.) are utilized after dispersion into TE buffer $(10 \mathrm{mM}$ Tris- $\mathrm{HCl}$, $1 \mathrm{mM}$ EDTA). Their $\zeta$-potentials were measured using Zetasizer Nano ZS (Malvern Panalytical Ltd., Zetasizer software ver. 7.12). For each particle, we obtained the values of $-73.8 \mathrm{mV}$ and $-68.6 \mathrm{mV}$, respectively. Note that these oversized particles are not capable of translocating through a $600 \mathrm{~nm}$ pore.

\section{Results and discussions}

As shown in Fig. 1a, the principle of a nanopore trapping method is based on a physical blocking of ion transport through a pore channel. The presence or absence of the particles at the pore can be checked by monitoring temporal changes in $I_{\text {ion }}$ : when a particle is captured, the current rapidly decreases due to a partial blocking of ion transport through the pore. In trapping, a particle is floating at a vicinity of the channel as the result of balance between electrophoretic force and drag force of electroosmotic flow. Both of these forces are proportional to the amplitude of voltage. The resistance of nanopore system can be described as a sum of two elements; $R=R_{\text {pore }}+2 R_{\text {acc }}$. In this equation, $R_{\text {pore }}=4 \rho L / \pi d_{\text {pore }}^{2}$ and $R_{\text {acc }}=\rho / 2 d_{\text {pore }}$ with electrical resistivity $\rho$, pore diameter $d_{\text {pore }}$, and thickness $L$ are pore resistance which means the component from inside of a pore and access resistance from electrodes to pore orifice, respectively [23-28]. In the measurement of $I_{\text {ion }}$ using a low aspect ratio nanopore, the factor of $L / d_{\text {pore }}^{2}$ in $R_{\text {pore }}$ would approach to zero and the total resistance could be approximated as $R_{\text {acc }}$. Therefore, the amplitude of current blockades in trapping strongly concerns with the volume and the surface charge density of entering particles.

Typical ionic current traces during a repetitive nanopore trapping is shown in Fig. 2a and focused in Fig. 2b. Before trapping, the pore is fully open and the current values represent a constant ionic current $I_{\text {open. }}$. When a particle is captured, the flow of ions is suppressed and the drop to $I_{\text {trap }}$ is observed $[16,17,29]$. As complete sealing should lead $I_{\text {ion }}$ to zero, the non-zero $I_{\text {trap }}$ indicates a particle floating at the vicinity of the pore because of the drag force of electroosmotic back flow antagonizing to the electrophoretic forces of the negatively-charged particle. After trapping, the particle can be released by a simple inversion of $V_{\mathrm{b}}$. The consecutive traces indicate long-term stability and reproducibility of the trapping/ detrapping processes.

Figure 3a shows voltage-dependence of $I_{\text {ion }}$ in the course of trapping processes for the both particles. At every $V_{\mathrm{b}}$ conditions tested, bimodal distributions corresponding to $I_{\text {open }}$ and $I_{\text {trap }}$ were obtained. To evaluate the dependence of ionic current suppressions on the voltage conditions, the conductance $G_{\text {trap }}=I_{\text {trap }} / V_{\mathrm{b}}$ was derived for the peak value in Fig. 3a. The voltage-dependency of $G_{\text {trap }}$ for each particle is shown Fig. 3b. Despite of the similarity at $V_{\mathrm{b}}=0.2$ and $0.3 \mathrm{~V}$. The displacement is observed at $V_{\mathrm{b}}=0.1$ and $0.4 \mathrm{~V}$ indicating further suppression by the particles sized $900 \mathrm{~nm}$. Assuming that linear relationship that higher (lower) voltage provide stronger (weaker) electrophoretical withdrawal of particles, this trend is counterintuitive. This diremption could be explained by contribution of electroosmotic flow. In 

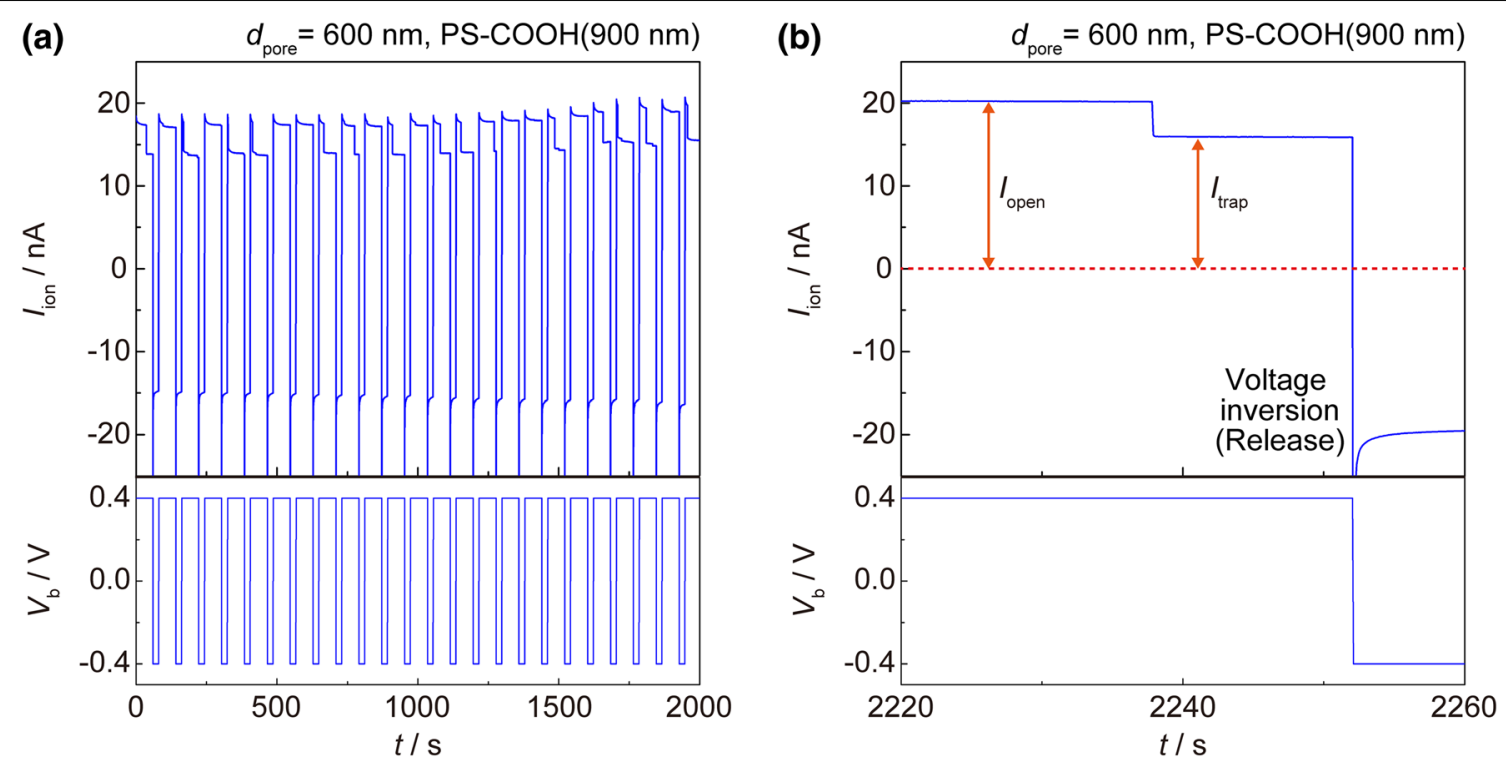

Fig. 2 a Typical consecutive current traces of electrophoretic particle trap and $\mathbf{b}$ magnified view of a single trace showing open pore current $I_{\text {open }}$ and suppressed $I_{\text {trap }}$. Trapping/detrapping processes are controlled by inversion of $V_{\mathrm{b}}$
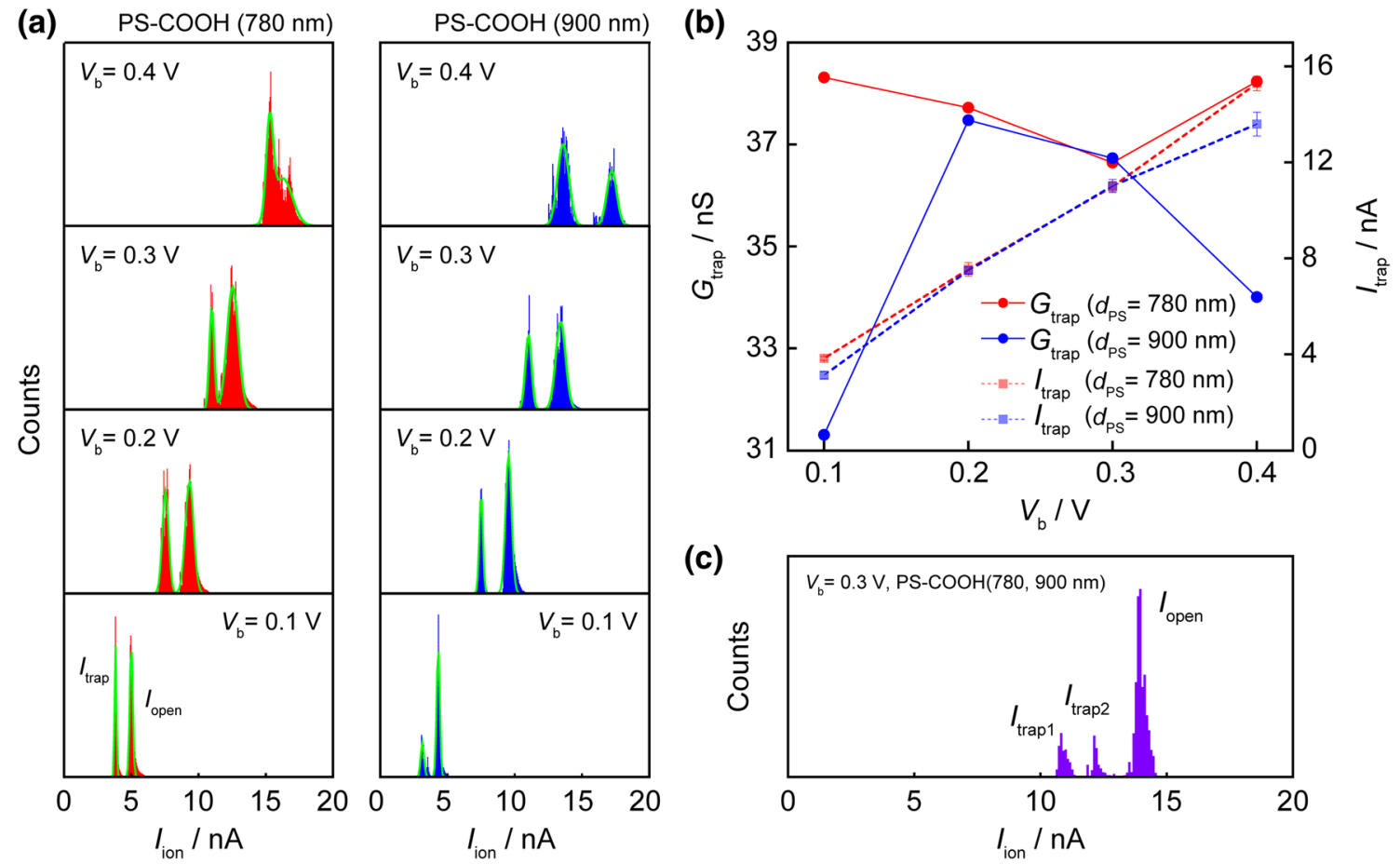

Fig. 3 Voltage dependence of $\mathbf{a}$ current histogram, $\mathbf{b}$ occlusion amplitude of single-sized particles. c Current distribution of multiple particles trapping

trapping, the particles is at the vicinity of a pore orifice sufficiently under electrophoretic force. However, at the same time, it enables effective application of dragging force of electroosmosis. This exception suggests a shift of the equilibrium between the two counteractive forces. Consequently, values of $I_{\text {trap }}$ has close relationship with 
the position of the trapped particle. In the quantitative approach of our previous work [16], the order of these forces are nearly equal and electrophoretic force is little larger. Hence, electroosmotic flow affect the particle position certainly. However, nanopore trapping system based on the force-balance is too complicated and further studies are needed in order to shed light on the detailed estimations. Besides, we also adopted a nanopore trapping method to mixture of these particles (Fig. 3c). From current traces, a trimodal histogram was obtained. Each peak can be ascribable to $I_{\text {open, }}, I_{\text {trap } 1}$ by the $780 \mathrm{~nm}$-sized particle, and $I_{\text {trap2 }}$ that of $900 \mathrm{~nm}$-sized one. For assignment, $I_{\text {trap }}$ was utilized from each value at $V_{\mathrm{b}}=0.3 \mathrm{~V}$. As a result, the two values, $10.89 \pm 0.20$ and $12.17 \pm 0.09 \mathrm{nA}$, were acquired as $I_{\text {trap } 1}$ and $I_{\text {trap2 }}$, respectively. Compared with the $I_{\text {trap }}$ values of the individual particle measurements in Fig. $\left.3 \mathrm{~b}, I_{\operatorname{trap}(780 \mathrm{~nm}}\right)=10.99 \pm 0.21 \mathrm{nA}$ and $I_{\text {trap }(900}$ $\mathrm{nm})=11.02 \pm 0.27 \mathrm{nA}$ and its potential of particles discrimination is confirmed. The results prove the possibility of discrimination of captured objects from their volume and surface charge properties.

To shed light on the relationship between $I_{\text {trap }}$ and positions of a particle, multiphysics simulation was conducted (Fig. 4 and see Additional file 1) [16, 17]. We approximate the pore-particle distance $(\Delta d)$ by comparisons between the experimental $I_{\text {trap }}$ with simulated ones. Interestingly, we found that under different $V_{\mathrm{b}}$, the trajectories of particles are quite different. Specifically, in both cases, it seems that $\Delta d$ tends to decrease with the voltage. It is inspiring that the shapes of voltage dependency of $\Delta d$ is similar with that of $G_{\text {trap }}$. However, in the same manner as $G_{\text {trap }}$, the displacement also appears in $\Delta d$ at the $V_{\mathrm{b}}=0.2$ and $0.3 \mathrm{~V}$. In other words, these particles show similar ionic suppression despite of deeper incursion of the particle sized $780 \mathrm{~nm}$. It seems that $900 \mathrm{~nm}$ sized particle cannot invade into a pore as $780 \mathrm{~nm}$ due to stronger application of electroosmotic flow, whereas lower curvature occupies transport pass for ions more effectively. It demonstrates ionic transports inhibited equally as a result of commensuration between contribution of particle curvatures and positions.

For further evaluation of relationship between the amplitude of ionic current suppression and blocking factor; volume and surface charge density, we applied nanopore trapping method to various particles $\left(d_{\mathrm{PS}}=0.78\right.$, $0.90 \mu \mathrm{m}$ (Thermofisher Scientific Inc.) and 0.79, $0.99 \mu \mathrm{m}$ (Polyscience, Inc.). If the surface charge density is the dominant factor of ionic current suppression, $0.99 \mu \mathrm{m}$ PS-COOH ( $($-potential: $-81.9 \mathrm{mV})$ should show the smallest $I_{\text {trap. }}$. However, the comparison showing in Additional file 1: Figure S2 reveals smaller particle which has relatively weak charge $\left(d_{\mathrm{PS}}=0.78\right.$ and $\left.0.79 \mu \mathrm{m}\right)$ can block ionic flow more effectively. This means that the certain superior of volume in ionic blockade. In addition, we employed a smaller pore sized $300 \mathrm{~nm}$ to trap two particles of $d_{\mathrm{PS}}=0.49$ and $0.52 \mu \mathrm{m}$ ( $\zeta$-potentials: -61.4 and $-62.7 \mathrm{mV}$, respectively) in $0.4 \times$ PBS buffer (Additional file 1: Figure S3a). Despite the similarity of their $\zeta$-potentials, the smaller particle demonstrates larger suppression. This result proves our assumption; the dominant cause of target volume on trapping current. Furthermore, we also attempted to capture the large particles $\left(d_{\mathrm{PS}}=0.78,0.79,0.90\right.$, and $\left.0.99 \mu \mathrm{m}\right)$ using this smaller pore (Additional file 1: Figure S3b). In spite of

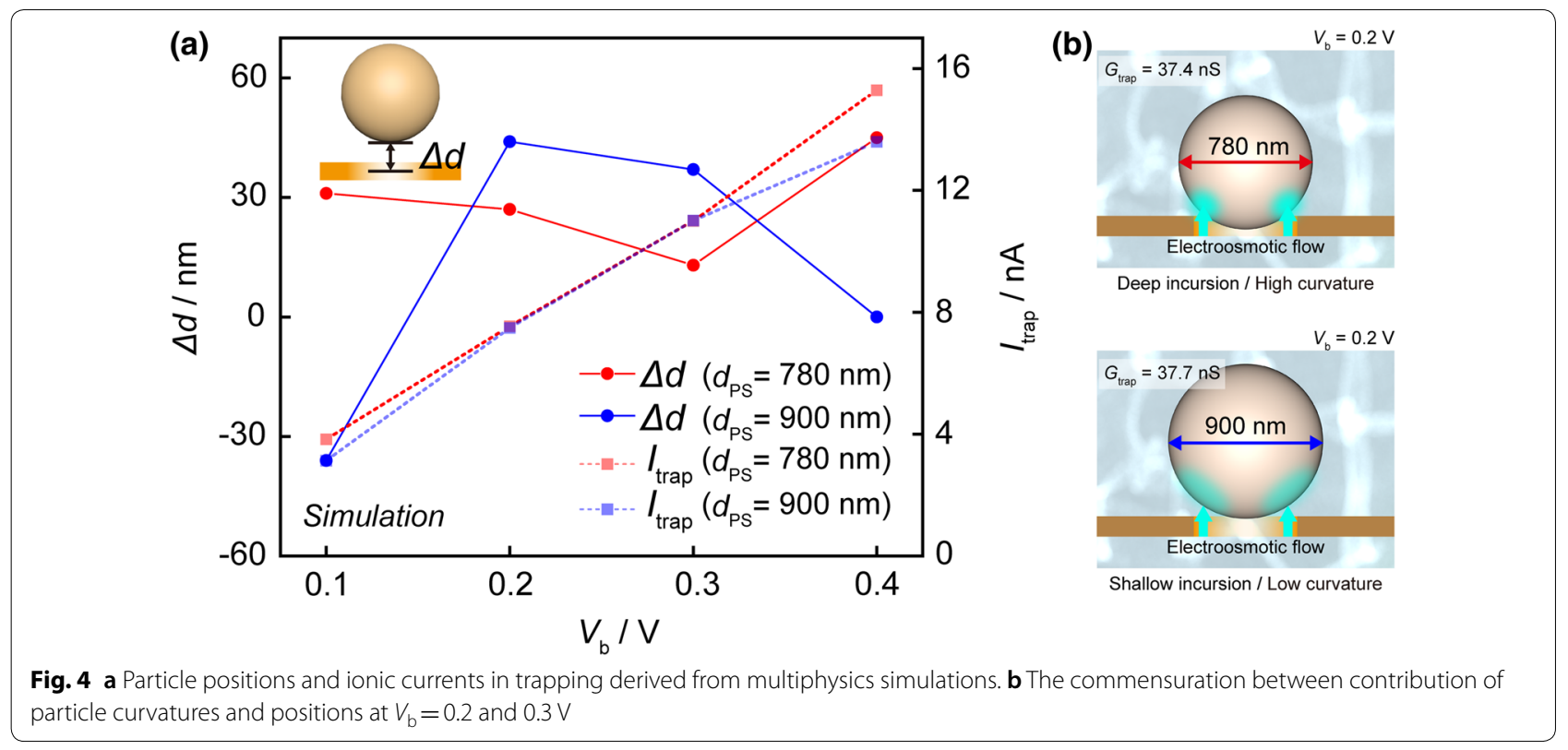


the larger $\zeta$-potentials than two particles trapped steady by this pore, the ionic currents in capturing shows great fluctuations indicating an incomplete immobilization since the effective electrical field is smaller and the contribution of electroosmotic force become large relatively. It reveals that the limitation of nanopore trapping is depend on the size of analyte.

\section{Conclusion}

In conclusion, it was revealed that a nanopore trapping method has ability of volume-specific discrimination with similarity of surface charge. In the particle trapping process using a pore, the factor determining ionic suppression is mixing of surface charge and particle size and their priority would appear in the similarity of another. The amplitude of ionic flow is reflected a particle properties of analytes representing a volume and the usefulness as status diagnostic method for single-particle is demonstrated. Besides, contradistinction between similarity of ionic blockade and dissimilarity of trajectory indicated detail electrokinetic factor in nanoscale. This result also suggests the possibility to serve as position modulator of micro-nano scale by a simple control of applied voltage in liquid condition and provide extensibility of sensing in such conditions.

\section{Additional file}

Additional file 1. Multiphysics simulation of particle blocking events, Distribution of ionic current in trappings of various particles, and Particle trapping employing a smaller nanopore.

\section{Authors' contributions}

MTs and MTa planned and designed the experiments. AA performed the experiments and analysed the data. AA and MTs co-wrote paper. All the authors discussed the data and reviewed the final manuscript. All authors read and approved the final manuscript.

\section{Acknowledgements}

We thank Prof. Yuhui He for his help in the multiphysics simulations.

\section{Competing interests}

The authors declare that they have no competing interests.

\section{Availability of data and materials}

All data generated or analysed during this study are included in this published article [and its additional file].

\section{Consent for publication \\ Not applicable.}

Ethics approval and consent to participate Not applicable.
Funding

This work was supported by Grant-in-Aid for JSPS fellows Grant Number 15J05282 and Grant-in-Aid for Young Scientists (B) Grant Number 17K14098.

\section{Publisher's Note}

Springer Nature remains neutral with regard to jurisdictional claims in published maps and institutional affiliations.

Received: 15 June 2018 Accepted: 5 March 2019

Published online: 14 March 2019

\section{References}

1. Luo L, German SR, Lan W-J, Holden DA, Mega TL, White HS. Resistivepulse analysis of nanoparticles. Annu Rev Anal Chem. 2014;7:513-35.

2. Yusko EC, Bruhn BR, Eggenberger OM, Houghtaling J, Rollings RC, Walsh NC, Nandivada S, Pindrus M, Hall AR, Sept D, Li J, Kalonia DS, Mayer M. Real-time shape approximation and fingerprinting of single proteins using a nanopore. Nat Nanotechnol. 2016;12:360-7.

3. Howorka S, Siwy Z. Nanopore analytics: sensing of single molecules. Chem Soc Rev. 2009;38:2360-84.

4. Miles BN, Ivanov AP, Wilson KA, Doğan F, Japrung D, Edel JB. Single molecule sensing with solid-state nanopores: novel materials, methods, and applications. Chem Rev Soc. 2013;42:15-28.

5. Tsutsui M, Yoshida T, Yokota K, Yasaki H, Yasui T, Arima A, Tonomura W, Nagashima K, Yanagida T, Kaji N, Taniguchi M, Washio T, Baba Y, Kawai T. Discriminating single-bacterial shape using low-aspect-ratio pores. Sci Rep. 2017;7:17371.

6. Tsutsui M, Tanaka M, Marui T, Yokota K, Yoshida T, Arima A, Tonomura W, Taniguchi M, Washio T, Okochi M, Kawai T. Identification of individual bacterial cells through the intermolecular interactions with peptidefunctionalized solid-state pores. Anal Chem. 2018;90:1511-5.

7. Ryuzaki S, Tsutsui M, He Y, Yokota K, Arima A, Morikawa T, Taniguchi M, Kawai T. Rapid structural analysis of nanomaterials in aqueous solutions. Nanotechnology. 2017;28:155501.

8. Arima A, Tsutsui M, Taniguchi M. Discriminating single-bacterial shape using low-aspect-ratio pores. Appl Phys Lett. 2014;104:163112.

9. Venkatesan BM, Bashir R. Nanopore sensors for nucleic acid analysis. Nat Nanotechnol. 2011;6:615-24.

10. Robertson JWF, Rodriques CG, Stanford VM, Rubinson KA, Krasilnikov OV, Kasianowicz JJ. Single-molecule mass spectrometry in solution using a solitary nanopore. Proc Natl Acad Sci USA. 2007;104:8207-11.

11. Reiner JE, Kasianowicz JJ, Nablo BJ, Robertson JWF. Theory for polymer analysis using nanopore-based single-molecule mass spectrometry. Proc Natl Acad Sci USA. 2010;107:12080-5.

12. Smeets RMM, Keyser UF, Krapf D, Wu M-Y, Dekker NH, Dekker C. Salt dependence of ion transport and DNA translocation through solid-state nanopores. Nano Lett. 2006;6:89-95.

13. Guan W, Joseph S, Park JH, Krstić PS, Reed MA. Paul trapping of charged particles in aqueous solution. Proc Natl Acad Sci USA. 2011;108:9326-30.

14. Keyser UF, van der Does J, Dekker C, Dekker NH. Optical tweezers for force measurements on DNA in nanopores. Rev Sci Instrum. 2006;77:105105.

15. Peng $H$, Ling XS. Reverse DNA translocation through a solid-state nanopore by magnetic tweezers. Nanotechnology. 2009;20:185101.

16. Tsutsui M, Maeda Y, He Y, Hongo S, Ryuzaki S, Kawano S, Kawai T, Taniguchi M. Trapping and identifying single-nanoparticles using a low-aspectratio nanopore. Appl Phys Lett. 2013;103:013108.

17. Arima A, Tsutsui M, He Y, Ryuzaki S, Taniguchi M. Electrical trapping mechanism of single-microparticles in a pore sensor. AIP Adv. 2016;6:115004.

18. Coulter WH. Means for counting particles suspended in a fluid. US Patent. 1953;2656506.

19. Yokota K, Tsutsui M, Taniguchi M. Electrode-embedded nanopores for label-free single-molecule sequencing by electric currents. RSC Adv. 2014;4:15886-99.

20. Chang S, He J, Kibel A, Lee M, Sankey O, Zhang P, Lindsay S. Tunnelling readout of hydrogen-bonding-based recognition. Nat Nanotechnol. 2009:4:297-301.

21. Tsutsui M, Taniguchi M, Yokota K, Kawai T. Identifying single nucleotides by tunnelling current. Nat Nanotechnol. 2010;5:286-90. 
22. Reed MA, Zhou C, Muller CJ, Burgin TP, Tour JM. Conductance of a molecular junction. Science. 1997;278:252-4.

23. Hall JE. Access resistance of a small circular pore. J Gen Physiol. 1975;66:531-2.

24. Tsutsui M, Hongo S, He Y, Taniguchi M, Gemma N, Kawai T. Singlenanoparticle detection using a low-aspect-ratio pore. ACS Nano. 2012;6:3499-505.

25. Davenport M, Healy K, Pevarnik M, Teslich N, Cabrini S, Morrison AP, Siwy ZS, Létant SE. The role of pore geometry in single nanoparticle detection. ACS Nano. 2012;6:8366-80.

26. Clarke J, Wu H-C, Jayasinghe L, Patel A, Reid S, Bayley H. Continuous base identification for single-molecule nanopore DNA sequencing. Nat Nanotechnol. 2009;4:265-70.
27. Wanunu M, Dadosh T, Ray V, Jin J, McReynolds L, Drndić M. Rapid electronic detection of probe-specific microRNAs using thin nanopore sensors. Nat Nanotechnol. 2010;5:807-14.

28. Kowalczyk SW, Grosberg AY, Rabin Y, Dekker C. Modeling the conductance and DNA blockade of solid-state nanopores. Nanotechnology. 2011;22:315101.

29. German SR, Luo L, White HS, Mega TL. Controlling nanoparticle dynamics in conical nanopores. J Phys Chem C. 2013;117:703-11.
Ready to submit your research? Choose BMC and benefit from:

- fast, convenient online submission

- thorough peer review by experienced researchers in your field

- rapid publication on acceptance

- support for research data, including large and complex data types

- gold Open Access which fosters wider collaboration and increased citations

- maximum visibility for your research: over $100 \mathrm{M}$ website views per year

At BMC, research is always in progress.

Learn more biomedcentral.com/submissions 